TRANSACTIONS OF THE

AMERICAN MATHEMATICAL SOCIETY

Volume 351, Number 5, Pages 1871-1894

S 0002-9947(99)02244-8

Article electronically published on January 26, 1999

\title{
SIMPLE FAMILIES OF THUE INEQUALITIES
}

\author{
GÜNTER LETTL, ATTILA PETHÖ, AND PAUL VOUTIER
}

\begin{abstract}
We use the hypergeometric method to solve three families of Thue inequalities of degree 3,4 and 6, respectively, each of which is parametrized by an integral parameter. We obtain bounds for the solutions, which are astonishingly small compared to similar results which use estimates of linear forms in logarithms.
\end{abstract}

\section{INTRODUCTION}

Although it is nowadays a routine matter to solve a single Thue equation, it was not until 1990 that a parametrized family of Thue equations was solved at one stroke by E. Thomas ([23]) and subsequently by other authors (see [14], [18], [16], [10]). In all these papers, linear forms of logarithms played a crucial role.

In the early 1960's, A. Baker [1] used the hypergeometric method, which goes back to A. Thue and C.L. Siegel, to obtain the first effective irrationality measure for algebraic numbers of degree at least three. Later, Chudnovsky [5] determined the precise asymptotic behaviour of the quantities which arise in this method. In this way, he was able to obtain much better effective irrationality measures for a wider class of algebraic numbers. He did not determine explicitly the constants involved. This was done shortly afterwards by Easton [7], whose results have recently been refined by Bennett [2] and Voutier [24] working independently.

Apart from A. Baker, who used his results to solve the Thue equation $X^{3}-2 Y^{3}=$ $n$ for $0 \leq n \leq 1000$ (unpublished thesis, Cambridge, 1965), this method was not used to determine all solutions of a given Thue equation until the work of Jian Hua Chen [3]. Recently, Chen and Voutier [4] have applied the hypergeometric method to give the complete solution of the family of Thue equations $F_{t}^{(4)}(X, Y)= \pm 1$ for $t \geq 128$ (see the notations below). Applying this method, one does not have to investigate the arithmetic of the underlying number field any more, e.g. neither fundamental units nor a description of the elements with given norm is needed. When this method does work, it almost always yields far better numerical results than using lower bounds for linear forms in logarithms.

Received by the editors March 31, 1997.

1991 Mathematics Subject Classification. Primary 11J25, 11J82; Secondary 11D25, $11 D 41$.

Research of the first author was supported by the Hungarian-Austrian governmental scientific and technological cooperation.

Research of the second author was supported by the Hungarian National Foundation for Scientific Research Grant No. 16791/95.

(C)1999 American Mathematical Society 
In the present paper we apply the hypergeometric method to solve the following three "simple" families of Thue inequalities:

$$
\left|F_{t}^{(j)}(X, Y)\right| \leq k(t),
$$

where $t \in \mathbb{Z} \backslash T_{j}, j \in\{3,4,6\}, k: \mathbb{Z} \rightarrow \mathbb{N}$ and

$$
\begin{aligned}
F_{t}^{(3)}(X, Y)= & X^{3}-t X^{2} Y-(t+3) X Y^{2}-Y^{3} \\
F_{t}^{(4)}(X, Y)= & X^{4}-t X^{3} Y-6 X^{2} Y^{2}+t X Y^{3}+Y^{4} \\
F_{t}^{(6)}(X, Y)= & X^{6}-2 t X^{5} Y-(5 t+15) X^{4} Y^{2} \\
& -20 X^{3} Y^{3}+5 t X^{2} Y^{4}+(2 t+6) X Y^{5}+Y^{6} .
\end{aligned}
$$

Here $T_{j}$ denotes a relatively small set of parameter values, for which this method does not apply, e.g. $T_{3}=\{t \in \mathbb{Z} \mid-32 \leq t \leq 29\}$. This is not caused by the incapacity of the authors, but by an intrinsic feature of the hypergeometric method. Through more elaborate computations in the proof of Proposition 2, one could replace the $T_{j}$ 's by slightly smaller sets, but these sets would never be empty, as one realizes from the asymptotic results in [5].

Let us remark that the families $F_{t}^{(3)}(X, Y)= \pm 1$ and $F_{t}^{(4)}(X, Y)=c$ with $c \in\{ \pm 1, \pm 4\}$ were completely solved in [14], [10] and [4]. For families of Thue equations of degree 6 there are - to our knowledge - no results up to now.

As usual, we call a solution $(x, y) \in \mathbb{Z}^{2}$ of a Thue inequality primitive if $\operatorname{gcd}(x, y)=1$, and $(0,0)$ is called the trivial solution. Since $F_{t}^{(j)}(u x, u y)=$ $u^{j} F_{t}^{(j)}(x, y)$, we will only consider primitive solutions.

The forms $F_{t}^{(j)}(X, Y)$ have non-trivial automorphisms, which produce from each solution an orbit of solutions. Therefore it suffices to find all solutions $(x, y) \in \mathbb{Z}$ with $\frac{y}{2}<x \leq y$ (or $|x| \leq y$ ), as will be explained in Lemmas 2, 9 and 10 .

From the theorems of this paper we will deduce the following particular results:

Corollary 1. For $t \geq 89$, the only primitive solutions $(x, y) \in \mathbb{Z}^{2}$ of

$$
\left|F_{t}^{(6)}(x, y)\right| \leq 120 t+323
$$

with $-\frac{y}{2}<x \leq y$ are $(0,1),(1,1),(1,2),(-1,3)$.

Corollary 2. For $t \geq 58$, the only primitive solutions $(x, y) \in \mathbb{Z}^{2}$ of

$$
\left|F_{t}^{(4)}(x, y)\right| \leq 6 t+7
$$

with $|x| \leq y$ are $(0,1),( \pm 1,1),( \pm 1,2)$.

Corollary 3. For $t \geq 30$ let $(x, y) \in \mathbb{Z}^{2}$ be a primitive solution of

$$
\left|F_{t}^{(3)}(x, y)\right| \leq k(t)
$$

with $\frac{8 k(t)}{2 t+3} \leq y$ and $-\frac{y}{2}<x \leq y$. Then we have

$$
|y|<0.4(120 k(t))^{1+\varepsilon(t)} \quad \text { with } \quad \varepsilon(t)=\frac{2.14}{\log \left(t+\frac{3}{2}\right)-3.44} .
$$

The Thue inequality of Corollary 3 was investigated and, for $k(t)=2 t+3$, completely solved in [15] by using linear forms in logarithms. The power of the hypergeometric method can be seen by comparing Corollary 3 with Theorem 1 in 
[15]. For example, for $t=1649$ the first yields $|y|<635 k(t)^{1.54}$, whereas the latter gives $|y|<10^{46649} k(t)^{228}$.

From the proofs of our theorems one can easily obtain effective measures of irrationality for the roots $\beta$ of $F_{t}^{(j)}(X, 1), t \notin T_{j}$, of the form

$$
\left|\beta-\frac{p}{q}\right|>\frac{1}{c|t||q|^{2+\mathrm{O}\left((\log |t|)^{-1}\right)}}
$$

for any $(p, q) \in \mathbb{Z} \times \mathbb{N}$ and some constant $c$. For $t \rightarrow \infty$, this result approaches Roth's bound, whereas the results of Theorem 2 in [15] tend to Liouville's bound as it always happens when using linear forms in logarithms.

In the next section we will indicate our reasons for calling the above three families of forms simple and show that any Thue inequality arising from a simple form can be transformed to one of the above shape. In particular, any cubic Thue inequality $|F(X, Y)| \leq k^{\prime}$, for which $F(X, 1)$ has a cyclic Galois group, is equivalent to a Thue inequality $\left|F_{t_{0}}^{(3)}(X, Y)\right| \leq k_{0}$ and thus can be solved with Corollary 3, provided $t_{0} \notin T_{3}$ (see Section 2 for more details).

In Section 3 we will investigate $F_{t}^{(6)}$ in detail, and in Theorem 1 state our results about the family of Thue inequalities

$$
\left|F_{t}^{(6)}(X, Y)\right| \leq k(t)
$$

The hypergeometric method is presented in Section 4. Proposition 2 gives a careful analysis of numerator and denominator of the approximating polynomials, which enables us to apply the hypergeometric method close to its natural limitation (e.g. we can treat $F_{t}^{(4)}$ for $t \geq 58$, compared with $t \geq 128$ in [4]).

Section 5 contains the proofs of Theorem 1 and Corollary 1. Finally, in Sections 6 and 7 we will present and prove the results for the simple quartic and cubic families, respectively.

\section{Simple RATIONAL FORMS}

$$
\begin{aligned}
& \text { For } A=\left(\begin{array}{ll}
a & b \\
c & d
\end{array}\right) \in \mathrm{GL}_{2}(\mathbb{Q}) \text { and } F \in \mathbb{Q}[X, Y] \text { we put } \\
& \qquad F^{A}:=F(a X+b Y, c X+d Y) \in \mathbb{Q}[X, Y] .
\end{aligned}
$$

This defines an action of $\mathrm{GL}_{2}(\mathbb{Q})$ on $\mathbb{Q}[X, Y]$, in fact an isomorphism between $\mathrm{GL}_{2}(\mathbb{Q})$ and the group of homogeneous automorphisms of $\mathbb{Q}[X, Y]$.

We call forms $F, G \in \mathbb{Q}[X, Y]$ equivalent if there exist some $A \in \mathrm{GL}_{2}(\mathbb{Q})$ and $r \in \mathbb{Q}^{\times}$(i.e. $r \neq 0$ ) with $r G=F^{A}$. This obviously defines an equivalence relation on the set of all rational forms.

If $F, G \in \mathbb{Z}[X, Y]$ are equivalent forms with integer coefficients there is some $A \in \mathrm{GL}_{2}(\mathbb{Q})$ with integer entries and some $r \in \mathbb{Q}^{\times}$such that $r G=F^{A}$. Then for any $k^{\prime}>0$, any solution $\left(x^{\prime}, y^{\prime}\right) \in \mathbb{Z}^{2}$ of $\left|G\left(x^{\prime}, y^{\prime}\right)\right| \leq k^{\prime}$ can be obtained from some solution $(x, y) \in \mathbb{Z}^{2}$ of $|F(x, y)| \leq k$ with $k=|r| k^{\prime}$ by solving $x=a x^{\prime}+b y^{\prime}, y=c x^{\prime}+d y^{\prime}$. Therefore it suffices to solve the diophantine inequality $|F| \leq k$ with arbitrary $k$ for one $F$ in each class of equivalent forms.

Definition 1. A form $F \in \mathbb{Q}[X, Y]$ is called simple if $F$ is irreducible over $\mathbb{Q}$ with $\operatorname{deg}(F) \geq 3$ and if there exists some non-trivial $A \in \mathrm{PGL}_{2}(\mathbb{Q})=\mathrm{GL}_{2}(\mathbb{Q}) /$ $\mathbb{Q}^{\times}\left(\begin{array}{ll}1 & 0 \\ 0 & 1\end{array}\right)$ such that $\psi_{A}: z \mapsto A z:=\frac{a z+b}{c z+d}$ permutes the zeroes of the underlying polynomial $P=F(X, 1)$ transitively. Here $\left(\begin{array}{ll}a & b \\ c & d\end{array}\right) \in \mathrm{GL}_{2}(\mathbb{Q})$ represents $A$. 
Remarks. 1. Let $F$ be a simple form and $\psi_{A}$ permute the roots of $P$. Then there is some $r \in \mathbb{Q}^{\times}$with $F^{A}=r F$. For any $B \in \mathrm{GL}_{2}(\mathbb{Q}), F^{B}$ is also simple and $B^{-1} A B$ permutes the roots of $P^{B}:=F^{B}(X, 1)$.

2. Be aware that in Definition 1 we require $\psi_{A}$ to operate transitively on the roots of $P$. For example, $F=X^{4}-2 Y^{4}$ satisfies all conditions of Definiton 1 (with $\left.A=\left(\begin{array}{rr}-1 & 0 \\ 0 & 1\end{array}\right)\right)$ but this last one.

3. Definition 1 implies that the roots of $P$ generate a cyclic number field of degree $\operatorname{deg}(F)$, where the Galois action on the roots of $P$ is given by $\psi_{A}$. These fields are well-known and some of them are the so-called "simplest" number fields (see [22], [9], [8], Appendix of [21]).

4. It is easy to see that any irreducible form $F \in \mathbb{Q}[X, Y]$ of degree 3 , for which $P=F(X, 1)$ has a cyclic Galois group, is simple. Indeed, if $\beta^{\prime}$ is conjugated to a root $\beta$ of $P$, we can write $\beta^{\prime}=r_{0}+r_{1} \beta+r_{2} \beta^{2}$ with $r_{i} \in \mathbb{Q}$ and $r_{2} \neq 0$, and one can check that $r_{0}+r_{1} \beta+r_{2} \beta^{2}=\frac{a \beta+b}{c \beta+d}$ has solutions $(a, b, c, d) \in \mathbb{Q}^{4}$. Thus by Lemma 1.b) below, any cyclic cubic number field can be generated by the roots of $F_{t}^{(3)}(X, 1)$ for some $t \in \mathbb{Z}$.

5. A problem similar to Lemma 1.a) below is dealt with in [6].

\section{Lemma 1.}

a) Each non-trivial torsion element of $\mathrm{PGL}_{2}(\mathbb{Q})$ has order $2,3,4$ or 6 and is conjugated to some power of $\left(\begin{array}{ll}0 & 1 \\ c & 0\end{array}\right)$ or $\left(\begin{array}{cc}1 & -1 \\ 1 & m\end{array}\right)$ with $c \in \mathbb{Q}^{\times}, m \in\{0,1,2\}$.

b) Up to equivalence - the only simple forms in $\mathbb{Q}[X, Y]$ are

$$
\begin{array}{ll}
F_{t}^{(3)} & \text { with } t \in \mathbb{Z}, \\
F_{t}^{(4)} & \text { with } t \in \mathbb{Z} \backslash\{-3,0,3\}, \\
F_{t}^{(6)} & \text { with } t \in \mathbb{Z} \backslash\{-8,-3,0,5\} .
\end{array}
$$

Proof. a) Let $A \in \mathrm{GL}_{2}(\mathbb{Q})$ represent a non-trivial torsion element of $\mathrm{PGL}_{2}(\mathbb{Q})$ of order $j \geq 2$. Then $A^{j}=\left(\begin{array}{cc}r & 0 \\ 0 & r\end{array}\right)$ with $r=\lambda_{1}^{j}=\lambda_{2}^{j}$, where $\lambda_{1}, \lambda_{2}$ are the eigenvalues of $A$. Thus $\lambda_{1} / \lambda_{2}$ is a root of unity and one easily shows that its order must be $j$. Since $\lambda_{1} / \lambda_{2}$ belongs to a quadratic number field over $\mathbb{Q}$, only $j=2,3,4,6$ is possible. If $X^{2}-a X-b$ is the characteristic polynomial of $A$, we use Frobenius' normal form (see e.g. [12], Kap. IX, §4) to obtain $A \sim\left(\begin{array}{ll}0 & 1 \\ b & a\end{array}\right)$, If $a=0$, we obtain the torsion elements of order 2 . If $a \neq 0$, we change $A$ to $\frac{1}{a} A \sim\left(\begin{array}{ll}0 & 1 \\ c & 1\end{array}\right)$ with $c=\frac{b}{a^{2}}$. Thus we have only to check for which $c \in \mathbb{Q}$ and $j \leq 6$ we obtain $\left(\begin{array}{ll}0 & 1 \\ c & 1\end{array}\right)^{j} \in \mathbb{Q}^{\times}\left(\begin{array}{ll}1 & 0 \\ 0 & 1\end{array}\right)$, and this yields - up to conjugation - the elements as stated in the lemma.

b) Let $F \in \mathbb{Q}[X, Y]$ be a simple form and $A \in \mathrm{PGL}_{2}(\mathbb{Q})$ be such that $\psi_{A}$ permutes the roots of $P=F(X, 1)$ transitively. Since $P$ has degree $\operatorname{deg}(P) \geq 3$ and is irreducible, $A$ must be a torsion element of order $\operatorname{deg}(P)$.

Put $T_{3}=\left(\begin{array}{cc}1 & 1 \\ -1 & 0\end{array}\right)$, which is conjugated to $\left(\begin{array}{cc}1 & -1 \\ 1 & 0\end{array}\right), T_{4}=\left(\begin{array}{cc}1 & -1 \\ 1 & 1\end{array}\right)$ and $T_{6}=$ $\left(\begin{array}{cc}1 & -1 \\ 1 & 2\end{array}\right)$. One can calculate that for $j \in\{3,4,6\}, F_{t}^{(j)}=\prod_{i=1}^{j}\left(X-\psi_{T_{j}^{i}}(\beta) Y\right)$, where $t$ (resp. $2 t$ for $j=6$ ) equals the sum of the roots $\psi_{T_{j}^{i}}(\beta)(i=1, \ldots, j$ ).

The irreducibility of $F_{t}^{(j)}$ for the indicated values of $t$ can easily be verified for $j=3,4$, and is proved in [8], Prop. 3.3, for $j=6$. Be aware that our parameter $t$ corresponds to $\frac{t-6}{4}$ in Gras' notation. Remark 1 above concludes the proof. 


\section{The Simple FAMily of Degree 6}

In this section we investigate the Thue inequality

$$
\left|F_{t}^{(6)}\right| \leq k(t)
$$

where $k: \mathbb{Z} \rightarrow \mathbb{N}$ is a function with positive values. For $t \geq 89$ we derive bounds for the solutions of (2) and solve it in the special case $k(t)=120 t+323$. The reason for this special choice of $k(t)$ is that $F_{t}^{(6)}(1,2)=37+120 t$ and $F_{t}^{(6)}(-1,3)=-323-120 t$ are the least non-constant values of $\left|F_{t}^{(6)}\right|$, as the reader can verify. Furthermore we have $F_{t}^{(6)}(1,1)=-27$ and $F_{t}^{(6)}(0,1)=1$; thus Corollary 1 implies that the latter is the only primitive solution of $\left|F_{t}^{(6)}(X, Y)\right|=1$.

To solve the inequality also for the remaining values $t \in T_{6}$, one has to invoke the usual method of using linear forms of logarithms. This requires a detailed study of the underlying sextic number field and is the subject of [11].

First, let us collect some important transformation properties of $F_{t}^{(6)}$.

\section{Lemma 2.}

a) The following relations hold:

$$
\begin{gathered}
F_{t}^{(6)}(X-Y, X+2 Y)=-27 F_{t}^{(6)}(X, Y) \\
F_{t}^{(6)}(X, Y)=F_{t}^{(6)}(-Y, X+Y)=F_{t}^{(6)}(-X-Y, X)=F_{t}^{(6)}(-X,-Y), \\
F_{t}^{(6)}(Y, X)=F_{-t-3}^{(6)}(X, Y) .
\end{gathered}
$$

b) If $(x, y) \in \mathbb{Z}^{2}$ is a solution of (2), then every pair of the orbit

$$
\{(x, y),(-y, x+y),(-x-y, x),(-x,-y),(y,-x-y),(x+y,-x)\}
$$

is also a solution of (2). The set of all solutions of (2) for fixed $t$ and $k(t)$ is a disjoint union of such orbits.

c) If an orbit of solutions of (2) contains one primitive solution, then all solutions in this orbit are primitive.

d) Any orbit of non-trivial solutions of (2) contains a pair $(x, y)$ with $y>0$ and $-\frac{y}{2}<x \leq y$.

Proof. a) - c) These assertions are easy to verify. We only mention that $T=$ $\left(\begin{array}{cc}0 & -1 \\ 1 & 1\end{array}\right)=\frac{1}{3} T_{6}^{2}$ yields an automorphism of the form $F_{t}^{(6)}$, which also permutes the elements of each orbit of solutions.

d) Each orbit of non-trivial solutions of (2) contains an element $(x, y)$ with $y>0$. If $|x|>y$ then either $\left(x^{\prime}, y^{\prime}\right)=(-y, x+y)$ or $\left(x^{\prime}, y^{\prime}\right)=(x+y,-x)$ satisfies $\left|x^{\prime}\right| \leq y^{\prime}$ and $0<y^{\prime}$. If $-y^{\prime} \leq x^{\prime} \leq-\frac{y^{\prime}}{2}$, then $\left(x_{0}, y_{0}\right)=\left(x^{\prime}+y^{\prime},-x^{\prime}\right)$ is an element of the orbit, which has all required properties.

This lemma shows that to solve the Thue inequality (2) it is sufficient to consider only parameters $t \in \mathbb{Z}$ with $-1 \leq t \neq 0,5$ and to restrict to primitive solutions $(x, y) \in \mathbb{Z}^{2}$ with $-\frac{y}{2}<x \leq y$. All solutions are then obtained from the orbit of $(x, y)$ and multiples $(u x, u y)$, as long as $\left|F_{t}^{(6)}(x, y)\right| \leq \frac{k(t)}{u^{6}}$. 
Before stating our main results, let us fix a numbering of the roots of the underlying polynomial. We put

$$
\begin{aligned}
P:= & F_{t}^{(6)}(X, 1)=X^{6}-2 t X^{5}-(5 t+15) X^{4}-20 X^{3} \\
& +5 t X^{2}+(2 t+6) X+1=\prod_{i=1}^{6}\left(X-\beta_{i}\right)
\end{aligned}
$$

no longer carrying the parameter $t \in \mathbb{Z}$ explicitly in our notation. From the preceding section we know that the rational map $\psi_{T_{6}}: z \mapsto \frac{z-1}{z+2}$ permutes the roots $\beta_{i}$ of $P$.

\section{Lemma 3.}

a) For $t \geq 6$ the following estimations hold:

$$
\begin{aligned}
2 t+\frac{5}{2}+\frac{10}{3 t}<\beta_{1} & =\beta<2 t+\frac{5}{2}+\frac{35}{8 t}, \\
1-\frac{3}{2 t}+\frac{8}{3 t^{2}}<\beta_{2} & =\frac{\beta-1}{\beta+2}<1-\frac{3}{2 t}+\frac{27}{8 t^{2}}, \\
-\frac{1}{2 t}+\frac{7}{8 t^{2}}-\frac{1}{t^{3}}<\beta_{3} & =\frac{-1}{\beta+1}<-\frac{1}{2 t}+\frac{7}{8 t^{2}}, \\
-\frac{1}{2}-\frac{3}{8 t}+\frac{1}{2 t^{2}}<\beta_{4} & =-\frac{\beta+2}{2 \beta+1}<-\frac{1}{2}-\frac{3}{8 t}+\frac{9}{16 t^{2}}, \\
-1-\frac{1}{2 t}+\frac{3}{5 t^{2}}<\beta_{5} & =-\frac{\beta+1}{\beta}<-1-\frac{1}{2 t}+\frac{11}{16 t^{2}}, \\
-2-\frac{3}{2 t}+\frac{9}{8 t^{2}}<\beta_{6} & =-\frac{2 \beta+1}{\beta-1}<-2-\frac{3}{2 t}+\frac{4}{3 t^{2}} .
\end{aligned}
$$

b) For $m \in\{2,3\}$ the continued fraction expansions of $\beta_{m}$ are

$$
\begin{gathered}
\beta_{2}=\left[0 ; 1,\left[\frac{2 t+1}{3}\right], \ldots\right] \quad \text { for } t \geq 6, \\
\beta_{3}=[-1 ; 1,2 t+2,1, \ldots] \text { for } t \geq 8 .
\end{gathered}
$$

Proof. a) One checks that $P$ changes its sign between the indicated bounds for each $\beta_{i}$.

b) If $\beta=\left[a_{0} ; a_{1}, a_{2}, \ldots\right]$ is the continued fraction expansion of some real number $\beta$ the $a_{i}$ 's are determined by the property that $\beta$ lies between $\left[a_{0} ; a_{1}, \ldots, a_{i}\right]$ and $\left[a_{0} ; a_{1}, \ldots, a_{i}+1\right]$. For $t \geq 6$, the bounds for $\beta_{m}$ from part a) immediately yield the claimed values for $a_{0}$ and $a_{1}$. For $t \geq 8$ we have $0+\frac{1}{1+\frac{3}{2 t-1}}<\beta_{2}<0+$ $\frac{1}{1+\frac{3}{2 t+2}}$, since $P$ changes its sign between these bounds. Thus $\frac{2 t-1}{3} \leq a_{2}<\frac{2 t+2}{3}$, which implies $a_{2}=\left[\frac{2 t+1}{3}\right]$. For $t=6,7$ we verified $a_{2}=\left[\frac{2 t+1}{3}\right]$ by direct calculation. The proof for $\beta_{3}$ runs in the same way.

Theorem 1. Let $t \geq 6$ and assume that $(x, y) \in \mathbb{Z}^{2}$ is a primitive solution of (2) with $-\frac{y}{2}<x \leq y$ and $1.572 \sqrt[4]{\frac{k(t)}{t}} \leq y$.

a) Then $\frac{x}{y}$ is a convergent to $\beta_{2}$ or $\beta_{3}$, and we have either $y=1$ or

$$
\left|\frac{x}{y}-\beta_{2}\right|<\frac{k(t)}{y^{6}(18 t-27)} \quad \text { and } \quad y \geq \frac{2 t+2}{3}
$$


or

$$
\left|\frac{x}{y}-\beta_{3}\right|<\frac{k(t)}{y^{6}(2 t+1)} \quad \text { and } \quad y \geq 2 t+3 .
$$

b) Assume $t \geq 89$ and put

$$
\kappa=\frac{\log \left(\sqrt{t^{2}+3 t+9}\right)+2.56}{\log \left(t+\frac{3}{2}\right)-3.09}<1+\frac{5.66}{\log \left(t+\frac{3}{2}\right)-3.09} .
$$

If $\frac{x}{y}$ is a convergent to $\beta_{2}$ we have

$$
y^{5-\kappa}<\frac{3.57 k(t)}{7.5^{\kappa}}
$$

If $\frac{x}{y}$ is a convergent to $\beta_{3}$ we have

$$
y^{5-\kappa}<\frac{31.41 k(t)}{12.99^{\kappa}} .
$$

Remarks. 1. From (5) and (6) one obtains upper bounds for $y$ only if $\kappa<5$, which happens for $t \geq 89$. These bounds have the form

$$
y<(N(t) k(t))^{\varepsilon(t)}
$$

which decrease very rapidly. Some numerical examples are shown in the following table:

\begin{tabular}{r|ccccccc}
$t$ & 89 & 90 & 100 & 150 & 200 & 1000 & $\infty$ \\
\hline$\varepsilon(t)$ & 128.81 & 25.96 & 3.26 & 0.93 & 0.69 & 0.40 & 0.25 \\
$N(t)$ for $(5)$ & $1.5 \cdot 10^{-4}$ & $1.6 \cdot 10^{-4}$ & $2.8 \cdot 10^{-4}$ & 0.0013 & 0.0028 & 0.024 & 0.476 \\
$N(t)$ for $(6)$ & $8.7 \cdot 10^{-5}$ & $9.4 \cdot 10^{-5}$ & $1.9 \cdot 10^{-4}$ & 0.0013 & 0.0035 & 0.054 & 2.42
\end{tabular}

2. Playing the lower bound for $|y|$ off against the upper bound in Theorem 1 , one can show that for $t \geq 1642$ any primitive solution $(x, y) \in \mathbb{Z}^{2}$ of

$$
\left|F_{t}^{(6)}(x, y)\right| \leq t^{3}
$$

with $-\frac{y}{2}<x \leq y$ must satisfy $y<1.572 \sqrt{t}$.

\section{The HYPERGEOMETRIC METHOD}

In this section we will establish notations and collect all the results which we will need to apply the hypergeometric method for solving our Thue inequalities. For references about the history of this method we refer to [5]. We will also use most notations as introduced in [4], only for some polynomials we use boldface or calligraphic letters to avoid ambiguity.

For $n, r \in \mathbb{N}$ put

$\mathcal{X}_{n, r}={ }_{2} \mathcal{F}_{1}\left(-r,-r-\frac{1}{n}, 1-\frac{1}{n}, X\right) \in \mathbb{Q}[X] \quad$ and $\quad \mathcal{X}_{n, r}^{*}=Y^{r} \mathcal{X}_{n, r}\left(\frac{X}{Y}\right) \in \mathbb{Q}[X, Y]$.

Here ${ }_{2} \mathcal{F}_{1}$ denotes the classical hypergeometric function, and thus $\mathcal{X}_{n, r}$ and $\mathcal{X}_{n, r}^{*}$ are rational polynomials of degree $r$.

The following proposition is a version of Thue's "Fundamentaltheorem" together with its relation to the hypergeometric function, as discovered by Siegel (see Lemma 2.1 in [4]; we substituted and simplified the expressions of [4] to obtain (8) below). 
Proposition 1. Let $P \in \mathbb{Q}[X]$ be a polynomial of degree $n \geq 2$ and assume that there is a quadratic polynomial $U \in \mathbb{Q}[X]$ with non-vanishing discriminant $\operatorname{disc}(U)$ such that

$$
U P^{\prime \prime}-(n-1) U^{\prime} P^{\prime}+\frac{n(n-1)}{2} U^{\prime \prime} P=0
$$

holds, where the prime denotes differentiation with respect to $X$. We put $Y_{1}=$ $2 U P^{\prime}-n U^{\prime} P$ and $\lambda=\frac{1}{4} \operatorname{disc}(U)$, and define the following polynomials:

$$
\begin{array}{rlrl}
\mathbf{a} & =\frac{n^{2}-1}{6}\left(\sqrt{\lambda} U^{\prime}+2 \lambda\right), & \mathbf{c} & =\frac{n^{2}-1}{6}\left(\sqrt{\lambda}\left(U^{\prime} X-2 U\right)+2 \lambda X\right), \\
\mathbf{b}=\frac{n^{2}-1}{6}\left(\sqrt{\lambda} U^{\prime}-2 \lambda\right), & \mathbf{d}=\frac{n^{2}-1}{6}\left(\sqrt{\lambda}\left(U^{\prime} X-2 U\right)-2 \lambda X\right), \\
\mathbf{z}=\frac{1}{2}\left(\frac{Y_{1}}{2 n \sqrt{\lambda}}+P\right), & \mathbf{u}=\mathbf{z}-P, \quad \mathbf{w}=\frac{\mathbf{z}}{\mathbf{u}} .
\end{array}
$$

Then for $r \in \mathbb{N}$ there are rational polynomials $A_{r}, B_{r} \in \mathbb{Q}[X]$ given by

$$
\begin{aligned}
& (\sqrt{\lambda})^{r} A_{r}=\mathbf{a} \mathcal{X}_{n, r}^{*}(\mathbf{z}, \mathbf{u})-\mathbf{b} \mathcal{X}_{n, r}^{*}(\mathbf{u}, \mathbf{z}), \\
& (\sqrt{\lambda})^{r} B_{r}=\mathbf{c} \mathcal{X}_{n, r}^{*}(\mathbf{z}, \mathbf{u})-\mathbf{d} \mathcal{X}_{n, r}^{*}(\mathbf{u}, \mathbf{z})
\end{aligned}
$$

such that for any root $\beta$ of $P$ the polynomial

$$
C_{r}=\beta A_{r}-B_{r}
$$

is divisible by $(X-\beta)^{2 r+1}$.

The following lemma collects the results of Lemmas 2.3, 2.5 and 2.6 of [4]. For roots of complex numbers we will agree to choose that number whose argument has minimal absolute value (and is positive if there is ambiguity); i.e. in the lemma below we have $-\frac{\pi}{n}<\arg \left(\mathbf{w}(\xi)^{1 / n}\right) \leq \frac{\pi}{n}$.

Lemma 4. Let the notations be as in Proposition 1.

a) For any $0 \neq \xi \in \mathbb{C}$ such that $w=\mathbf{w}(\xi)$ is not a negative real number or zero,

$$
\begin{aligned}
(\sqrt{\lambda})^{r} C_{r}(\xi)= & \left(\beta\left(\mathbf{a}(\xi) \mathbf{w}(\xi)^{1 / n}-\mathbf{b}(\xi)\right)-\left(\mathbf{c}(\xi) \mathbf{w}(\xi)^{1 / n}-\mathbf{d}(\xi)\right)\right) \mathcal{X}_{n, r}^{*}(\mathbf{u}(\xi), \mathbf{z}(\xi)) \\
& -(\beta \mathbf{a}(\xi)-\mathbf{c}(\xi)) \mathbf{u}(\xi)^{r} R_{n, r}(\mathbf{w}(\xi))
\end{aligned}
$$

with

$$
R_{n, r}(w)=\frac{\Gamma(r+1+1 / n)}{r ! \Gamma(1 / n)} \int_{1}^{w}(1-x)^{r}(x-w)^{r} x^{-(r+1-1 / n)} d x
$$

where the path of integration is the straight line from 1 to $w$.

b) Let $w=e^{i \varphi}$ with $0<\varphi<\pi$ and put $\sqrt{w}=e^{i \varphi / 2}$. Then

$$
\left|R_{n, r}(w)\right| \leq \frac{\Gamma(r+1+1 / n)}{\Gamma(1 / n) r !} \varphi|1-\sqrt{w}|^{2 r}
$$

and

$$
\left|\mathcal{X}_{n, r}(w)\right| \leq 4 \frac{\Gamma(1-1 / n) r !}{\Gamma(r+1-1 / n)}|1+\sqrt{w}|^{2 r-2}
$$


If we substitute for $X$ a rational number $\nu \in \mathbb{Q}$ lying near the root $\beta$, Proposition 1 provides us with two sequences $A_{r}(\nu), B_{r}(\nu) \quad(r \in \mathbb{N})$ of rational numbers from which to obtain very good rational approximations to the algebraic number $\beta$ we wish to consider. To get integer sequences, which are necessary for applying Lemma 7 below, we simply clear the denominators. However, we will proceed somewhat carefully as there are benefits to be obtained from sharp estimates for these denominators.

Let $\Delta_{n, r}$ denote the smallest positive integer such that $\Delta_{n, r} \mathcal{X}_{n, r}^{*}(X, Y) \in$ $\mathbb{Z}[X, Y]$. Chudnovsky [5], Section 4 , has determined the asymptotic behaviour of $\Delta_{n, r}$ as $r \rightarrow \infty$. In [24], one of us used the ideas contained in Chudnovsky's proof to determine precise information about the prime divisors of $\Delta_{n, r}$ and their multiplicities. The following lemma is a corollary to Proposition 1 of [24]. We denote the set of all rational primes with $\mathbb{P}$.

Lemma 5. Let $n \in\{3,4,6\}, r \in \mathbb{N}$ and $p \in \mathbb{P}$ with $p \mid \Delta_{n, r}$.

a) We have

$$
p \equiv-1 \bmod (n) \quad \text { and } \quad v_{p}\left(\Delta_{n, r}\right) \leq\left[\frac{\log (n r)}{2 \log p}+\frac{1}{2}\right],
$$

where $v_{p}(m)$ denotes the exponent to which $p$ divides $m \in \mathbb{Z} \backslash\{0\}$. b) If $p \geq \sqrt[3]{n r}$ then $p^{2} \nmid \Delta_{n, r}$ and there exists an $A \in \mathbb{N} \cup\{0\}$ with

$$
\frac{n r}{n A+n-1}<p \leq \frac{n r}{n A+1} .
$$

To obtain upper bounds for $\Delta_{n, r}$ from this lemma, we shall need some analytic estimates. Fortunately for our work, Ramaré and Rumely [19] have recently determined sharp estimates of the type required here. For $x \in \mathbb{R}$, let $\pi(x)$ be the number of primes less than or equal to $x$ and $\theta(x ; k, l)=\sum_{\substack{p \in \mathbb{P}, p \leq x \\ p \equiv l \bmod (k)}} \log p$.

Lemma 6. a) For $x>1$, we have $\pi(x)<1.26 \frac{x}{\log x}$.

b) For $n \in\{3,4,6\}$ and $x \geq 3800$, we have $0.491 x<\theta(x ; n, n-1)<0.505 x$.

Proof. a) This is Corollary 2 of [20]. b) Since $1.7982 \sqrt{x}<0.005 x<0.009 x$ for $x \geq 130000$, from the entries for $k=3,4$ and 6 in Tables 1 and 2 of [19], the stated inequalities for $\theta(x ; n, n-1)$ hold for such $x$. It is a straightforward matter to calculate $\theta(x ; n, n-1)$ for $x<130000$. These calculations demonstrate that part b) is true.

The following proposition will prove more useful than just bounding $\Delta_{n, r}$ from above.

Proposition 2. Let $r \in \mathbb{N}, \mu_{n}=\prod_{p \in \mathbb{P}, p \mid n} p^{1 /(p-1)} \quad$ and $N_{n, r} \quad$ be the greatest common divisor of the numerators of the coefficients of $\mathcal{X}_{n, r}\left(1-n \mu_{n} X\right)$.

a) $N_{n, r}$ is divisible by $n^{r} \cdot \prod_{p \in \mathbb{P}, p \mid n} p^{v_{p}(r !)}$.

b) We have

$$
\frac{4^{r} \Delta_{3, r}}{N_{3, r}} \frac{\Gamma(2 / 3) r !}{\Gamma(r+2 / 3)}<1.9 e^{0.83 r} \quad \text { and } \quad\left(\frac{27}{4}\right)^{r} \frac{\Delta_{3, r}}{N_{3, r}} \frac{\Gamma(r+4 / 3)}{\Gamma(1 / 3) r !}<0.87 e^{1.3 r} .
$$


c) We have

$$
\frac{4^{r} \Delta_{4, r}}{N_{4, r}} \frac{\Gamma(3 / 4) r !}{\Gamma(r+3 / 4)}<0.83 e^{0.99 r} \quad \text { and } \quad \frac{16^{r} \Delta_{4, r}}{N_{4, r}} \frac{\Gamma(r+5 / 4)}{\Gamma(1 / 4) r !}<0.2 e^{2.37 r} .
$$

d) We have

$$
\frac{16^{r} \Delta_{6, r}}{N_{6, r}} \frac{\Gamma(5 / 6) r !}{\Gamma(r+5 / 6)}<1.2 e^{2.56 r} \quad \text { and } \quad \frac{27^{r} \Delta_{6, r}}{N_{6, r}} \frac{\Gamma(r+7 / 6)}{\Gamma(1 / 6) r !}<0.16 e^{3.09 r} .
$$

Proof. a) This is Proposition 5.1 of [5].

b) It is easy to calculate $\mathcal{X}_{3, r}$ and the other relevant quantities for small $r$. Note that to find $N_{3, r}$, we used equations (5.2)-(5.4) of [5]. We performed the required computations for each $1 \leq r \leq 1000$ and showed that the stated inequalities hold for all $r$ in this range. This took about 430 seconds. ${ }^{1}$ We nearly get equality between the terms in the first inequality at $r=4$ and it is from consideration of this value of $r$ that we select the constant 1.9. The constant 0.87 , in the second inequality, arises in the same way from the values of the terms in that inequality for $r=10$.

For $r>1000$, direct calculation of the quantities themselves soon becomes prohibitive, but we are not yet able to obtain good analytic estimates. So, at this point, we combine calculation and some elementary analysis to show that our inequalities hold for intermediate-sized $r$ 's.

Let us first estimate $\frac{4^{r}}{N_{3, r}} \frac{\Gamma(2 / 3) r !}{\Gamma(r+2 / 3)}$. From part a), we know that $3^{r+v_{3}(r !)} \leq$ $N_{3, r}$. Moreover, one can show that for $r \in \mathbb{N}$ and $p \in \mathbb{P}$ one has

$$
v_{p}(r !) \geq \frac{r}{p-1}-\frac{\log (r+1)}{\log p} .
$$

Therefore, $N_{3, r} \geq \frac{3^{3 r / 2}}{r+1}$. Next,

$$
\frac{\Gamma(2 / 3) r !}{\Gamma(r+2 / 3)}=\frac{3}{2} \prod_{i=2}^{r} \frac{i}{i-1 / 3}<\frac{3}{2} \exp \left(\int_{1}^{r} \log \frac{x}{x-1 / 3} d x\right)<\left(\frac{3 e r}{2}\right)^{1 / 3},
$$

for $r \geq 1$. Therefore,

$$
\frac{4^{r}}{N_{3, r}} \frac{\Gamma(2 / 3) r !}{\Gamma(r+2 / 3)}<3.2 r^{4 / 3}\left(\frac{4}{3 \sqrt{3}}\right)^{r} .
$$

We now consider $\left(\frac{27}{4}\right)^{r} \frac{1}{N_{3, r}} \frac{\Gamma(r+4 / 3)}{\Gamma(1 / 3) r !}$. From before, we have $\frac{3^{3 r / 2}}{r+1} \leq N_{3, r}$. We also estimate the $\Gamma$-factors as above, finding that

$$
\frac{\Gamma(r+4 / 3)}{\Gamma(1 / 3) r !}=\frac{4}{9} \prod_{i=2}^{r} \frac{i+1 / 3}{i}<\frac{4}{9} \exp \left(\int_{1}^{r} \log \frac{x+1 / 3}{x} d x\right)<\frac{1}{3}(e r)^{1 / 3} .
$$

\footnotetext{
${ }^{1}$ The computation mentioned above as well as the others mentioned in this section were performed using the number-theoretic software package UBASIC 8.74 on an IBM-PC compatible with an Intel 80486DX2 running at $66 \mathrm{MHz}$. Some of these calculations were also checked using Maple V Release 3 on the same machine as well as, occasionally, by hand. In no case were any discrepancies found.
} 
Combining these estimates, we obtain

$$
\left(\frac{27}{4}\right)^{r} \frac{1}{N_{3, r}} \frac{\Gamma(r+4 / 3)}{\Gamma(1 / 3) r !}<r^{4 / 3}\left(\frac{3 \sqrt{3}}{4}\right)^{r} .
$$

To estimate $\Delta_{3, r}$, we turn to Lemma 5 . Let $\Delta_{3, r \text { small }}$ be the contribution to $\Delta_{3, r}$ from those primes less than $\sqrt[3]{3 r}$ and let $\Delta_{3, r, \text { large }}$ be the contribution from the larger primes. From Lemma 5.a), we know that

$$
\Delta_{3, r, \text { small }}<\prod_{\substack{p<(3 r)^{1 / 3} \\ p \equiv 2 \bmod (3)}}(3 r)^{1 / 2} p^{1 / 2}<\exp \left(\frac{\pi(\sqrt[3]{3 r}) \log (3 r)+\theta(\sqrt[3]{3 r} ; 3,2)}{2}\right) .
$$

Using our upper bound for $\theta(x ; 3,2)$ from Lemma 6.b) and a direct calculation of $\theta(x ; 3,2)$ for all $x<3800$, we find that $\theta(x ; 3,2)<0.5094 x$ for all $x>0$. This is also Theorem 5.1 of [13]. Combining this with our upper bound for $\pi(x)$ in Lemma 6.a), we obtain

$$
\Delta_{3, r, \text { small }}<\exp (3.2 \sqrt[3]{r}) .
$$

From Lemma 5.b), we see that

$$
\Delta_{3, r, \text { large }} \leq \exp \left(\sum_{A=0}^{N}\left(\theta\left(\frac{3 r}{3 A+1} ; 3,2\right)-\theta\left(\frac{3 r}{3 A+2} ; 3,2\right)\right)\right),
$$

where $N$ is the largest positive integer for which $\frac{3 r}{3 N+1}>(3 r)^{1 / 3}$. We can easily calculate this quantity directly, which we do for each $1000<r \leq 20000$, a computation taking about 5250 seconds. Combining the estimates we obtain in this way with the bounds in (14), (15) and (16), we determined upper bounds for the quantities in question for each $1000<r \leq 20000$, and always found that the inequalities in part b) hold. This concludes the proof for such $r$.

Finally, we turn to the case $r>20000$. From (16), we obtain

$$
\Delta_{3, r, \text { small }}<e^{0.0044 r},
$$

so it remains to consider $\Delta_{3, r \text {, large }}$. For any positive integer $N$, we have

$$
\Delta_{3, r, \text { large }} \leq \exp \left(\sum_{A=0}^{N} \theta\left(\frac{3 r}{3 A+1} ; 3,2\right)-\sum_{A=0}^{N-1} \theta\left(\frac{3 r}{3 A+2} ; 3,2\right)\right) .
$$

Since $r>20000$, each of the quantities $\frac{3 r}{3 A+1}$ for $A=0, \ldots, 4$ and $\frac{3 r}{3 A+2}$ for $A=0, \ldots, 3$ are greater 3800 . So choosing $N=4$, we have

$$
\Delta_{3, r, \text { large }} \leq \exp \left(\sum_{A=0}^{4} 0.505 \frac{3 r}{3 A+1}-\sum_{A=0}^{3} 0.491 \frac{3 r}{3 A+2}\right)<e^{1.03 r},
$$

from Lemma 6.b). Combining this inequality with (17) yields $\Delta_{3, r}<e^{1.0344 r}$. Now we just use the fact that $3.2 r^{4 / 3}<e^{0.00072 r}, \log \left(\frac{4}{3 \sqrt{3}}\right)=-0.26162 \ldots$, along with (14) and (15) to complete the proof of part b) of the proposition.

c) We proceed in the exact same way here as in the proof of part b). A direct calculation of the relevant quantities for each $1 \leq r \leq 1000$ demonstrates that this part of the proposition holds for all such $r$. The constants 0.83 and 0.2 are obtained from $r=3$. 
As above, we have

$$
\frac{4^{r}}{N_{4, r}} \frac{\Gamma(3 / 4) r !}{\Gamma(r+3 / 4)}<2.76 \frac{r^{5 / 4}}{2^{r}}, \quad \frac{16^{r}}{N_{4, r}} \frac{\Gamma(r+5 / 4)}{\Gamma(1 / 4) r !}<0.63 r^{5 / 4} 2^{r}
$$

and

$$
\Delta_{4, r, \text { small }}<\exp (3.43 \sqrt[3]{r})
$$

where $\Delta_{4, r, \text { small }}$ is the contribution to $\Delta_{4, r}$ from those primes less than $\sqrt[3]{4 r}$ and $\Delta_{4, r, \text { large }}$ the contribution from the larger primes. We use the fact that $\theta(x ; 4,3)<0.529 x$ for all $x>0$, which we can easily obtain from Lemma 6.b) with the help of a little computation, along with Lemma 6.a) to prove this last inequality.

We estimate $\Delta_{4, r, \text { large }}$ from above as before for $1000<r \leq 20000$. Our bounds show that part c) of the proposition holds for such $r$.

For larger $r$, we also proceed as above using $N=5$ this time. This yields $\Delta_{4, r, \text { large }}<e^{1.6708 r}$ for these $r$. Combining all these estimates completes the proof of this part of the proposition.

d) Again, we prove this part of the lemma in the same way as we proved part b). The constants 1.2 and 0.16 both arise from $r=30$. We find that

$$
\frac{16^{r}}{N_{6, r}} \frac{\Gamma(5 / 6) r !}{\Gamma(r+5 / 6)} \leq 4.8 r^{13 / 6}\left(\frac{4}{3 \sqrt{3}}\right)^{r}, \quad \frac{27^{r}}{N_{6, r}} \frac{\Gamma(r+7 / 6)}{\Gamma(1 / 6) r !}<0.8 r^{13 / 6}\left(\frac{3 \sqrt{3}}{4}\right)^{r},
$$

and

$$
\Delta_{6, r, \text { small }}<\exp (3.9 \sqrt[3]{r})
$$

Here $\Delta_{6, r, \text { small }}$ is the contribution to $\Delta_{6, r}$ from those primes less than $\sqrt[3]{6 r}$ and $\Delta_{6, r, \text { large }}$ the contribution from the larger primes. We can show that $\theta(x ; 6,5)<$ $0.509 x$ for all $x>0$, a fact which we use as before to establish the inequality for $\Delta_{6, r \text { small }}$.

We estimate $\Delta_{6, r, \text { large }}$ from above for $1000<r \leq 20000$ by means of computation as in the case $n=3$. Combining the estimates found in this way with the bounds above, we determine that part d) of the proposition holds for such $r$.

For larger $r$, we once more use the ideas described for the case $n=3$, this time choosing $N=5$. This yields $\Delta_{6, r, \text { large }}<e^{2.8144 r}$ for these $r$. We apply all these estimates again to complete the proof of this part of the proposition.

Finally, we need a result showing how good rational approximations to a real number $\theta$ yield an explicit bound for the measure of irrationality of $\theta$. For this purpose we cite Lemma 2.8 of [4]:

Lemma 7. Let $\theta \in \mathbb{R}$. Suppose that there are real numbers $k_{0}, l_{0}>0$ and $E, Q>1$ such that for all $n \in \mathbb{N}$ there exist rational integers $p_{n}$ and $q_{n}$ with $\left|q_{n}\right|<k_{0} Q^{n}$ and $\left|q_{n} \theta-p_{n}\right| \leq l_{0} E^{-n}$ satisfying $p_{n} q_{n+1} \neq p_{n+1} q_{n}$. Then for any rational integers $p$ and $q$ with $|q| \geq \frac{1}{2 l_{0}}$, we have

$$
\left|\theta-\frac{p}{q}\right|>\frac{1}{c|q|^{\kappa+1}}, \quad \text { where } \quad c=2 k_{0} Q\left(2 l_{0} E\right)^{\kappa} \quad \text { and } \quad \kappa=\frac{\log Q}{\log E} .
$$




\section{Proofs of Theorem 1 and of Corollary 1}

Proof of Theorem 1. Let $t \geq 6$ and assume that $(x, y) \in \mathbb{Z}^{2}$ with $\operatorname{gcd}(x, y)=1$ and $-\frac{y}{2}<x \leq y$ a solution of (2). For $1 \leq i \leq 6$, put $\delta_{i}=\left|\frac{x}{y}-\beta_{i}\right|$ and let $m$ be defined by $\delta_{m}=\min \left\{\delta_{i} \mid 1 \leq i \leq 6\right\}$. Since $\frac{x}{y} \in\left(-\frac{1}{2}, 1\right]$, it is obvious from Lemma 3.a) that $m \in\{2,3,4\}$, and more precisely,

$$
\frac{x}{y} \in\left(-\frac{1}{2}, 1\right] \cap\left(\frac{\beta_{m}+\beta_{m+1}}{2}, \frac{\beta_{m}+\beta_{m-1}}{2}\right) .
$$

a) From $\left|F_{t}^{(6)}(x, y)\right|=\prod_{i=1}^{6}\left|x-\beta_{i} y\right|$, we obtain

$$
\delta_{m} \leq \frac{k(t)}{y^{6} \prod_{i \neq m} \delta_{i}}
$$

and using the fact that $\frac{x}{y}$ must be in the interval above along with the bounds for $\beta_{i}$ from Lemma 3.a) we arrive at

$$
\prod_{i \neq m} \delta_{i}>s_{1, m}= \begin{cases}\frac{15}{4} t-\frac{121}{32} & \text { for } m=2 \\ \frac{21}{64} t+\frac{37}{256} & \text { for } m=3 \\ \frac{15}{32} t+\frac{273}{256} & \text { for } m=4\end{cases}
$$

Thus we have $\delta_{m}<\frac{k(t)}{y^{6} s_{1, m}}$. It is well known that if $\delta_{m}<\frac{1}{2 y^{2}}$, then $\frac{x}{y}$ must be a convergent to $\beta_{m}$, and this holds whenever $y \geq 1.572 \sqrt[4]{\frac{k(t)}{t}}>\sqrt[4]{\frac{512 k(t)}{84 t+37}} \geq \sqrt[4]{\frac{2 k(t)}{s_{1, m}}}$.

The first two convergents of $\beta_{4}$ are -1 and $-\frac{1}{2}$, thus all the convergents of $\beta_{4}$ are less than or equal to $-\frac{1}{2}$, and therefore the case $m=4$ cannot occur.

From Lemma 3.b) we find that the first convergents of $\beta_{2}$ are $0,1,1-\frac{1}{a_{2}+1} \geq$ $1-\frac{3}{2 t+2}$. If $\frac{x}{y}$ is a convergent to $\beta_{2}$ with $y>1$ we thus have $\frac{2 t-1}{2 t+2} \leq \frac{x}{y} \leq 1$. Using the bounds for $\beta_{i}$ from Lemma 3.a) we obtain $\prod_{i \neq 2} \delta_{i}>s_{2,2}=18 t-27$.

Similarly, for $t \geq 8$ the convergents of $\beta_{3}$ are $-1,0,-\frac{1}{2 t+3},-\frac{1}{2 t+4}, \ldots$, and with $-\frac{1}{2 t+3} \leq \frac{x}{y} \leq-\frac{1}{2 t+4}$ we obtain $\prod_{i \neq 3} \delta_{i}>2 t+1$. For $t=6,7$, a direct calculation of the first convergents of $\beta_{3}$ shows that $-\frac{1}{2 t+4} \leq \frac{x}{y} \leq-\frac{1}{2 t+5}$, which again yields $\prod_{i \neq 3} \delta_{i}>2 t+1$. With (18) we thus obtain

$$
\delta_{m}<\frac{k(t)}{y^{6} s_{2, m}} \quad \text { with } \quad s_{2, m}= \begin{cases}18 t-27 & \text { for } m=2, \\ 2 t+1 & \text { for } m=3,\end{cases}
$$

and the claimed lower bounds for $y$ are given by the denominators of the convergents of $\beta_{m}$.

b) Choosing $U=X^{2}+X+1$, our polynomials $P$ from (3) satisfy the differential equation (7), and we may apply Proposition 1 . We have $\lambda=-\frac{3}{4}$, and (8) specializes to

$$
\begin{array}{ll}
\mathbf{a}=\frac{35}{6} \sqrt{-3}\left(X-\rho^{2}\right), \quad \mathbf{c}=\frac{35}{6} \sqrt{-3}(\rho X-1), \\
\mathbf{b}=\frac{35}{6} \sqrt{-3}(X-\rho)=-\overline{\mathbf{a}}, & \mathbf{d}=\frac{35}{6} \sqrt{-3}\left(\rho^{2} X-1\right)=-\overline{\mathbf{c}} .
\end{array}
$$

Here $\rho=\frac{-1+\sqrt{-3}}{2}$ and $\rho^{2}=\frac{-1-\sqrt{-3}}{2}$ are the third roots of unity and - denotes complex conjugation. 
To obtain good rational approximations for $\beta_{2}$ and $\beta_{3}$, we substitute $X=1$ and $X=0$, resp. Our first step is to show the following:

Let $r \in \mathbb{N}$ and put $M_{r, 0}=18^{r} \frac{2 \sqrt{3}}{35} \frac{\Delta_{6, r}}{N_{6, r}}$ and $M_{r, 1}=\left(\frac{2}{3}\right)^{r} \frac{2}{35} \frac{\Delta_{6, r}}{N_{6, r}}$. Then for $\nu=0,1$,

$$
p_{r}(\nu)=M_{r, \nu} B_{r}(\nu) \quad \text { and } \quad q_{r}(\nu)=M_{r, \nu} A_{r}(\nu)
$$

are rational integers.

Inserting $X=0$, we obtain from (9)

$$
\begin{gathered}
z:=t-3 \rho^{2}=3 \sqrt{-3} \mathbf{z}(0), \quad u:=t-3 \rho=3 \sqrt{-3} \mathbf{u}(0) \text { and } \\
w:=\frac{t-3 \rho^{2}}{t-3 \rho}=\mathbf{w}(0) .
\end{gathered}
$$

Let us remark that $u=\bar{z}$ are algebraic integers and $|u|=\sqrt{t^{2}+3 t+9}$. Using (10) and the above expressions for the quantities which occur there for $X=0$, we get

$$
\begin{aligned}
M_{r, 0} A_{r}(0)= & 18^{r} \frac{2 \sqrt{3}}{35} \frac{\Delta_{6, r}}{N_{6, r}}\left(\frac{2}{\sqrt{-3}}\right)^{r} \frac{35}{6} \sqrt{-3} \\
& \times\left(-\rho^{2} \mathcal{X}_{6, r}^{*}(\mathbf{z}(0), \mathbf{u}(0))+\rho \mathcal{X}_{6, r}^{*}(\mathbf{u}(0), \mathbf{z}(0))\right) \\
= & \frac{\Delta_{6, r}}{N_{6, r}}\left(\frac{36}{\sqrt{-3}}\right)^{r}(-i)\left(\rho^{2} \mathbf{u}(0)^{r} \mathcal{X}_{6, r}(w)-\rho \mathbf{z}(0)^{r} \mathcal{X}_{6, r}(\bar{w})\right) \\
= & \frac{\Delta_{6, r}}{N_{6, r}}(-i)\left(\rho^{2}(-4 u)^{r} \mathcal{X}_{6, r}(w)-\overline{\rho^{2}(-4 u)^{r} \mathcal{X}_{6, r}(w)}\right) \\
= & 2 \Im\left(\rho^{2}(-4 u)^{r} \frac{\Delta_{6, r}}{N_{6, r}} \mathcal{X}_{6, r}(w)\right) .
\end{aligned}
$$

Since $w=1-12 \sqrt{3} \frac{-i}{4 u}=1-6 \mu_{6} \frac{-i}{4 u}$ and $\frac{4 u}{-i}$ is an algebraic integer, using the definition of $\Delta_{6, r}$ and $N_{6, r}$ in the preceding section we see that $\rho^{2}(-4 u)^{r} \frac{\Delta_{6, r}}{N_{6, r}} \mathcal{X}_{6, r}(w)$ is an algebraic integer in $\mathbb{Q}(\rho)$. Therefore twice its imaginary part must be a rational integer.

For $X=1$ we obtain $\mathbf{z}(1)=-27 \mathbf{z}(0)=3 \sqrt{-3} z, \quad \mathbf{u}(1)=3 \sqrt{-3} u$ and $\mathbf{w}(1)=\frac{z}{u}=w$ with $z, u$ and $w$ as defined in (20). This time we obtain

$$
\begin{aligned}
M_{r, 1} A_{r}(1) & =\left(\frac{2}{3}\right)^{r} \frac{2}{35} \frac{\Delta_{6, r}}{N_{6, r}}\left(\frac{2}{\sqrt{-3}}\right)^{r} \frac{35}{2}\left(\rho \mathcal{X}_{6, r}^{*}(\mathbf{z}(1), \mathbf{u}(1))+\rho^{2} \mathcal{X}_{6, r}^{*}(\mathbf{u}(1), \mathbf{z}(1))\right) \\
& =\frac{\Delta_{6, r}}{N_{6, r}}\left(\rho(4 u)^{r} \mathcal{X}_{6, r}(w)+\overline{\rho(4 u)^{r} \mathcal{X}_{6, r}(w)}\right) \\
& =2 \Re\left(\rho(4 u)^{r} \frac{\Delta_{6, r}}{N_{6, r}} \mathcal{X}_{6, r}(w)\right),
\end{aligned}
$$

which is a rational integer by the same reasoning as above. The proofs for the integrality of $M_{r, \nu} B_{r}(\nu)$ are completely analogous. 
Now we derive upper bounds for $\left|M_{r, \nu} A_{r}(\nu)\right|$. Using (13) and Proposition 2.d) we obtain for $\nu=0,1$

$$
\begin{aligned}
\left|M_{r, \nu} A_{r}(\nu)\right| & \leq 2|4 u|^{r} \frac{\Delta_{6, r}}{N_{6, r}}\left|\mathcal{X}_{6, r}(w)\right| \\
& \leq 2\left(4 \sqrt{t^{2}+3 t+9}\right)^{r} \frac{\Delta_{6, r}}{N_{6, r}} 4 \frac{\Gamma(5 / 6) r !}{\Gamma(r+5 / 6)}|1+\sqrt{w}|^{2 r-2} \\
& <2\left(\sqrt{t^{2}+3 t+9}\right)^{r} 16^{r} \frac{\Delta_{6, r}}{N_{6, r}} \frac{\Gamma(5 / 6) r !}{\Gamma(r+5 / 6)}<2.4\left(e^{2.56} \sqrt{t^{2}+3 t+9}\right)^{r} .
\end{aligned}
$$

Our next step is to find an upper bound for $\left|M_{r, \nu} C_{r}(\nu)\right|$. We have $\sqrt{w}=$ $\frac{t-3 \rho^{2}}{\sqrt{t^{2}+3 t+9}}$ and derive the estimations

$$
\begin{gathered}
\arg (w) \leq \frac{\Im(w)}{\Re(w)} \leq \frac{3 \sqrt{3}}{t} \quad \text { and } \\
|u| \cdot|1-\sqrt{w}|^{2}=2 \sqrt{t^{2}+3 t+9}-(2 t+3) \leq \frac{27}{4(t+3 / 2)} .
\end{gathered}
$$

First we will show that the factor of $\mathcal{X}_{6, r}^{*}$ in (11) vanishes for $\nu=0,1$, i.e. that $\beta_{2}$ equals $\frac{\mathbf{c}(1) w^{1 / 6}-\mathbf{d}(1)}{\mathbf{a}(1) w^{1 / 6}-\mathbf{b}(1)}=\frac{\rho w^{1 / 6}+1}{w^{1 / 6}+\rho}$ and $\beta_{3}$ equals $\frac{\mathbf{c}(0) w^{1 / 6}-\mathbf{d}(0)}{\mathbf{a}(0) w^{1 / 6}-\mathbf{b}(0)}=$ $\frac{\rho w^{1 / 6}-\rho}{w^{1 / 6}-\rho^{2}}$. To show that $\frac{\rho w^{1 / 6}+1}{w^{1 / 6}+\rho}$ is a root of $P$, one uses $\rho^{2}+\rho+1=0$ and calculates that $F_{t}^{(6)}\left(\rho w^{1 / 6}+1, w^{1 / 6}+\rho\right)=(t-3 \rho)(-3-6 \rho) w+\left(-t+3 \rho^{2}\right)(-3-6 \rho)=$ 0 . One also verifies that $\frac{\rho w^{1 / 6}+1}{w^{1 / 6}+\rho}$ lies near 1 , thus $\beta_{2}=\frac{\rho w^{1 / 6}+1}{w^{1 / 6}+\rho}$. Using $\beta_{3}=\frac{\beta_{2}-1}{\beta_{2}+2}$, one easily concludes that $\beta_{3}=\frac{\rho w^{1 / 6}-\rho}{w^{1 / 6}-\rho^{2}}$.

Using (11) we calculate that for $\nu=0,1$ we have

$$
\left|M_{r, \nu} C_{r}(\nu)\right|=|4 u|^{r} \frac{\Delta_{6, r}}{N_{6, r}}\left|R_{6, r}(w)\right| \cdot \begin{cases}\sqrt{1+\beta_{3}+\beta_{3}^{2}} & \text { if } \nu=0, \\ \sqrt{1+\beta_{2}+\beta_{2}^{2}} & \text { if } \nu=1 .\end{cases}
$$

From Lemma 3.a) we derive that $\sqrt{1+\beta_{3}+\beta_{3}^{2}}<1$ and $\sqrt{1+\beta_{2}+\beta_{2}^{2}}<\sqrt{3}$. Using (12), (21) and Prop. 2.d) we obtain

$$
\left|M_{r, 0} C_{r}(0)\right|<\frac{3 \sqrt{3}}{t} 27^{r} \frac{\Delta_{6, r}}{N_{6, r}} \frac{\Gamma(r+7 / 6)}{\Gamma(1 / 6) r !}\left(\frac{1}{t+3 / 2}\right)^{r}<\frac{0.48 \sqrt{3}}{t}\left(\frac{e^{3.09}}{t+3 / 2}\right)^{r}
$$

and

$$
\left|M_{r, 1} C_{r}(1)\right|<\frac{9}{t} 27^{r} \frac{\Delta_{6, r}}{N_{6, r}} \frac{\Gamma(r+7 / 6)}{\Gamma(1 / 6) r !}\left(\frac{1}{t+3 / 2}\right)^{r}<\frac{1.44}{t}\left(\frac{e^{3.09}}{t+3 / 2}\right)^{r} .
$$

Summarizing, we obtained the following result: for $\nu=0,1$ and $r \in \mathbb{N}$ there are rational integers $p_{r}(\nu), q_{r}(\nu)$ with

$$
\begin{gathered}
\left|q_{r}(\nu)\right|<2.4\left(e^{2.56} \sqrt{t^{2}+3 t+9}\right)^{r} \\
\left|\beta_{3} q_{r}(0)-p_{r}(0)\right|<\frac{0.48 \sqrt{3}}{t}\left(\frac{e^{3.09}}{t+3 / 2}\right)^{r}
\end{gathered}
$$


and

$$
\left|\beta_{2} q_{r}(1)-p_{r}(1)\right|<\frac{1.44}{t}\left(\frac{e^{3.09}}{t+3 / 2}\right)^{r}
$$

With Lemma 2.7 of [4] we make sure that $p_{r}(\nu) q_{r+1}(\nu) \neq p_{r+1}(\nu) q_{r}(\nu)$, thus we may apply Lemma 7 with $k_{0}=2.4, Q=e^{2.56} \sqrt{t^{2}+3 t+9}, E=e^{-3.09}\left(t+\frac{3}{2}\right)$ and $l_{0}=\frac{0.48 \sqrt{3}}{t}$ or $\frac{1.44}{t}$, resp. Combining this with (19) we obtain, for any $(x, y)$ satisfying the conditions of Theorem 1.b) and with $\kappa$ defined by (4),

$$
\frac{1}{2 k_{0} Q\left(2 l_{0} E\right)^{\kappa} y^{\kappa+1}}<\left|\beta_{m}-\frac{x}{y}\right|<\frac{k(t)}{y^{6} s_{2, m}}
$$

and therefore

$$
y^{5-\kappa}<\frac{2 k_{0} Q k(t)}{\left(2 l_{0} E\right)^{-\kappa} s_{2, m}} .
$$

Inserting the numerical values for $m=2,3$ and making some easy estimations for $t \geq 89$ we obtain (5) and (6), and the proof of Theorem 1.b) is completed.

Remark. From the last part of the proof above one immediately obtains effective irrationality measures for the roots $\beta_{2}$ and $\beta_{3}$ of $P$ : for any $p, q \in \mathbb{Z}$ with $|q| \geq 0.61 t$ we have

$$
\left|\beta_{m}-\frac{p}{q}\right|>\frac{1}{8.5 t|q|^{2+\varepsilon(t)}} \quad \text { with } \quad \varepsilon(t)=\frac{5.66}{\log \left(t+\frac{3}{2}\right)-3.09} .
$$

Proof of Corollary 1. Assume that for some $t \geq 6, \quad(x, y) \in \mathbb{Z}^{2}$ is a primitive solution of $\left|F_{t}^{(6)}(x, y)\right| \leq 120 t+323$ with $-\frac{y}{2}<x \leq y$. Since $1.572 \sqrt[4]{120+\frac{323}{t}}<$ 6 , we may apply Theorem 1 for the case that $6 \leq y$. With the help of a computer we checked that (5) is in contradiction to $y \geq \frac{2 t+2}{3}$ for $t \geq 99$ and (6) contradicts $y \geq 2 t+3$ for $t \geq 89$. Above these bounds, any primitive solution must satisfy $-\frac{y}{2}<x \leq y \leq 5$, and checking the values of $F_{t}^{(6)}$ for these small arguments yields the asserted solutions of the Thue inequality.

It remains to consider the case when $89 \leq t \leq 98$ and $\frac{x}{y}$ is a convergent to $\beta_{2}$. From (5) we obtain upper bounds $y<y_{0}(t)$, and some values are given in the following table:

\begin{tabular}{c|cccc}
$t$ & 89 & 90 & 93 & 98 \\
\hline$y_{0}(t)$ & $8.6 \cdot 10^{28}$ & $4.4 \cdot 10^{6}$ & 540 & 73
\end{tabular}

Of course, an easy way to finish the proof of the corollary is to check all possible convergents with the help of a computer. Nevertheless, we will exhibit another idea (compare with Theorem 2 in [17]), which reduces the amount of computational work, and which we will also use in the next section.

Lemma 8. Let $\beta \in \mathbb{R}$ and $\beta=\left[a_{0} ; a_{1}, a_{2}, \ldots\right]$ be its continued fraction expansion with convergents $\frac{p_{j}}{q_{j}}$. For $0 \leq j_{0}<j_{1}$ put $A=\max \left\{a_{j} \mid j_{0} \leq j \leq j_{1}\right\}$. If for some $j$ with $j_{0} \leq j<j_{1}$ we have

$$
\left|\beta-\frac{p_{j}}{q_{j}}\right|<\frac{c}{q_{j}^{k}}
$$


with some $c>0$ and $k \geq 2$ then

$$
A>\frac{q_{j_{0}}^{k-2}}{c}-2
$$

Proof. By the theory of continued fractions and by the definition of $A$ we have

$$
\left|\beta-\frac{p_{j}}{q_{j}}\right|>\frac{1}{\left(a_{j+1}+2\right) q_{j}^{2}} \geq \frac{1}{(A+2) q_{j}^{2}} .
$$

Since the denominators of the convergents are an increasing sequence, we have on the other hand

$$
\left|\beta-\frac{p_{j}}{q_{j}}\right|<\frac{c}{q_{j}^{k}} \leq \frac{c}{q_{j}^{2} q_{j_{0}}^{k-2}} .
$$

Combining these inequalities we obtain the lower bound for $A$.

To finish the proof of Corollary 1, we apply Lemma 8 with $\beta=\beta_{2}, j_{0}=2$, $q_{2} \geq \frac{2 t+2}{3}, \quad k=6$ and $c=\frac{120 t+323}{18 t-27}$, using the results of Theorem 1.a). The existence of a further primitive solution would imply that $A>0.14\left(\frac{2 t+2}{3}\right)^{4} \geq$ 1814400 . Computing the continued fraction expansion of $\beta_{2}$ for any $89 \leq t \leq 98$ up to an index $j_{1}$ with $q_{j_{1}}>y_{0}(t)$, the largest partial quotient we found was 713 , the 18-th partial quotient of $\beta_{2}$ for $t=89$. Thus (2) has no further solutions.

\section{The SIMPLE FAMILY OF DEGREE 4}

Now we consider the Thue inequality

$$
\left|F_{t}^{(4)}\right| \leq k(t),
$$

where $k: \mathbb{Z} \rightarrow \mathbb{N}$. For $t \geq 58$ we will derive bounds for the solutions of (22) and solve it in the special case $k(t)=6 t+7$. For $t \in \mathbb{Z}$ let us put

$$
P:=F_{t}^{(4)}(X, 1)=X^{4}-t X^{3}-6 X^{2}+t X+1=\prod_{i=1}^{4}\left(X-\beta_{i}\right) .
$$

From Lemma 1.b) we know that $\psi_{T_{4}}: z \mapsto \frac{z-1}{z+1}$ permutes the roots $\beta_{i}$ of $P$.

Lemma 9 below collects information about $F_{t}^{(4)}$ and $P$, which is analogous to Lemmas 2 and 3. Most of these results can be found in [4] or [10].

\section{Lemma 9.}

a) The following relations hold:

$$
\begin{gathered}
F_{t}^{(4)}(X-Y, X+Y)=-4 F_{t}^{(4)}(X, Y), \\
F_{t}^{(4)}(X, Y)=F_{t}^{(4)}(Y,-X)=F_{t}^{(4)}(-X,-Y)=F_{t}^{(4)}(-Y, X)=F_{-t}^{(4)}(Y, X) .
\end{gathered}
$$

b) If $(x, y) \in \mathbb{Z}^{2}$ is a solution of (22), then every pair of the orbit

$$
\{(x, y),(y,-x),(-x,-y),(-y, x)\}
$$

is also a solution of (22). Any orbit of non-trivial solutions of (22) contains a pair $(x, y)$ with $y>0$ and $|x| \leq y$. 
c) For $t \neq 0, \pm 3, \quad P$ is irreducible over $\mathbb{Q}[X]$, and for $t \geq 6$ the roots of $P$ satisfy the following inequalities:

$$
\begin{aligned}
t+\frac{4}{t}<\beta_{1} & =\beta<t+\frac{5}{t}, \\
1-\frac{2}{t}+\frac{2}{t^{2}}<\beta_{2} & =\frac{\beta-1}{\beta+1}<1-\frac{2}{t}+\frac{3}{t^{2}}, \\
-\frac{1}{t}+\frac{4}{t^{3}}<\beta_{3} & =-\frac{1}{\beta}<-\frac{1}{t}+\frac{5}{t^{3}}, \\
-1-\frac{2}{t}-\frac{2}{t^{2}}<\beta_{4} & =-\frac{\beta+1}{\beta-1}<-1-\frac{2}{t} .
\end{aligned}
$$

d) For $m \in\{2,3\}$ the continued fraction expansions of $\beta_{m}$ are

$$
\begin{aligned}
& \beta_{2}= \begin{cases}{\left[0 ; 1, u-1,1,1,\left[\frac{u-3}{5}\right], \ldots\right]} & \text { if } t=2 u \geq 16 \text { is even, } \\
{\left[0 ; 1, u-1, a_{3}, \ldots\right] \text { with } a_{3} \geq \frac{t}{3}} & \text { if } t=2 u-1 \geq 3 \text { is odd, }\end{cases} \\
& \beta_{3}=\left[-1 ; 1, t-1,\left[\frac{t}{5}\right], \ldots\right] \text { if } t \geq 5 .
\end{aligned}
$$

Theorem 2. Let $t \geq 6$ and assume that $(x, y) \in \mathbb{Z}^{2}$ is a primitive solution of (22) with $|x| \leq y$ and $4 \sqrt{\frac{k(t)}{2 t-1}} \leq y$.

a) Then $\frac{x}{y}$ is a convergent to $\beta_{2}$ or $\beta_{3}$, and we have either $y=1$ or

$$
\left|\frac{x}{y}-\beta_{2}\right|<\frac{k(t)}{y^{4}(2 t-4)} \quad \text { and } \quad y \geq \frac{t}{2}
$$

or

$$
\left|\frac{x}{y}-\beta_{3}\right|<\frac{k(t)}{y^{4} t} \quad \text { and } \quad y \geq t .
$$

b) Assume $t \geq 58$ and put

$$
\kappa=\frac{\log \left(\sqrt{t^{2}+16}\right)+0.99}{\log t-2.37}<1+\frac{3.37}{\log t-2.37} .
$$

If $\frac{x}{y}$ is a convergent to $\beta_{2}$ then

$$
y^{3-\kappa}<\frac{3.28 k(t)}{2.97^{\kappa}} .
$$

If $\frac{x}{y}$ is a convergent to $\beta_{3}$ then

$$
y^{3-\kappa}<\frac{4.48 k(t)}{5.34^{\kappa}} .
$$

Proof of Theorem 2. a) The proof runs in complete analogy to that of Theorem 1, so we will just indicate some intermediate steps and leave the rest to the reader. Let $(x, y) \in \mathbb{Z}^{2}$ with $|x| \leq y$ be a primitive solution of (22) for some $t \geq 6$. Again we put $\delta_{i}=\left|\frac{x}{y}-\beta_{i}\right|$ and $\delta_{m}=\min \left\{\delta_{i} \mid 1 \leq i \leq 4\right\}$, thus

$$
\frac{x}{y} \in[-1,1] \cap\left(\frac{\beta_{m}+\beta_{m+1}}{2}, \frac{\beta_{m}+\beta_{m-1}}{2}\right),
$$


and we obtain

$$
\delta_{m}<\frac{k(t)}{y^{4} s_{1, m}} \quad \text { with } \quad \prod_{i \neq m} \delta_{i}>s_{1, m}= \begin{cases}\frac{3 t-5}{4} & \text { for } m=2, \\ \frac{2 t-1}{8} & \text { for } m=3 \\ \frac{6 t+7}{8} & \text { for } m=4\end{cases}
$$

If $y \geq 4 \sqrt{\frac{k(t)}{2 t-1}} \geq \sqrt{\frac{2 k(t)}{s_{1, m}}}$ holds, $\frac{x}{y}$ must be a convergent to $\beta_{m}$ for some $m \in\{2,3,4\}$. Since -1 is the largest convergent of $\beta_{4}$ and also a convergent of $\beta_{3}$, we may omit the case $m=4$.

If $\frac{x}{y}$ is a convergent to $\beta_{2}$ with $y>1$, Lemma 9.d) yields $\frac{t-2}{t} \leq \frac{x}{y} \leq 1$ (for even $t \leq 14$ we checked this separately), and using Lemma 9.c) we obtain $\prod_{i \neq 2} \delta_{i}>s_{2,2}=2 t-4$. If $\frac{x}{y}$ is a convergent to $\beta_{3}$ with $y>1$, we similarly deduce that $-\frac{1}{t} \leq \frac{x}{y} \leq-\frac{t-4}{t^{2}-4 t+5}$ and $\prod_{i \neq 3} \delta_{i}>s_{2,3}=t$ in this case. From these results, the validity of the claimed upper bounds for the $\delta_{m}$ 's follows, and an examination of the denominators of the second convergents of the $\beta_{m}$ 's yields the lower bounds for $y$.

b) (For more details of this proof we refer the reader to Section 3 of [4].) With $U=X^{2}+1$ we may apply Proposition 1 and get $\lambda=-1$,

$$
\begin{array}{ll}
\mathbf{a}=5(i X-1), & \mathbf{b}=5(i X+1)=-\overline{\mathbf{a}}, \\
\mathbf{c}=5(-X-i), & \mathbf{d}=5(X-i)=-\overline{\mathbf{c}} .
\end{array}
$$

We substitute $X=0$ and $X=1$ to get good rational approximations for $\beta_{3}$ and $\beta_{2}$, respectively. Defining

$$
M_{r, 0}=\frac{1}{10} 8^{r} \frac{\Delta_{4, r}}{N_{4, r}} \quad \text { and } \quad M_{r, 1}=\frac{1}{10} 2^{r} \frac{\Delta_{4, r}}{N_{4, r}},
$$

we obtain rational integers

$$
p_{r}(\nu)=M_{r, \nu} B_{r}(\nu) \quad \text { and } \quad q_{r}(\nu)=M_{r, \nu} A_{r}(\nu)
$$

where $r \in \mathbb{N}$ and $\nu=0,1$.

Let us give here the proof that $q_{r}(1)$ is an integer. The proofs of the integrality of $q_{r}(0)$ and $p_{r}(\nu)$ are completely analogous. Inserting $X=1$, we obtain from (9)

$$
z:=4-i t=-2 \mathbf{z}(1), \quad u:=4+i t=2 \mathbf{u}(1) \quad \text { and } \quad w:=-\frac{z}{u}=\mathbf{w}(1)
$$

with algebraic integers $u=\bar{z}$ and $|u|=\sqrt{t^{2}+16}$. Now (10) yields

$$
\begin{aligned}
M_{r, 1} A_{r}(1) & =\frac{1}{10} 2^{r} \frac{\Delta_{4, r}}{N_{4, r}}(-i)^{r}\left(5(i-1)\left(\frac{u}{2}\right)^{r} \mathcal{X}_{4, r}(w)-5(i+1)\left(-\frac{z}{2}\right)^{r} \mathcal{X}_{4, r}\left(w^{-1}\right)\right) \\
& =\frac{1}{2}(-i)^{r} \frac{\Delta_{4, r}}{N_{4, r}}\left((i-1) u^{r} \mathcal{X}_{4, r}(w)+(-1)^{r} \overline{(i-1) u^{r} \mathcal{X}_{4, r}(w)}\right)
\end{aligned}
$$

Since $w=1-8 \frac{1}{u}=1-4 \mu_{4} \frac{1}{u}$, it follows that $(i-1) \frac{\Delta_{4, r}}{N_{4, r}} u^{r} \mathcal{X}_{4, r}(w)$ is an algebraic integer in $\mathbb{Q}[i]$. Up to sign, the above expression for $M_{r, 1} A_{r}(1)$ is the real or imaginary part of this algebraic integer, thus a rational integer. 
Using (13) and Proposition 2.c) we obtain

$$
\begin{aligned}
\left|M_{r, 0} A_{r}(0)\right| & \leq \frac{\Delta_{4, r}}{N_{4, r}}\left|u^{r} \mathcal{X}_{4, r}(w)\right| \leq 4^{r} \frac{\Delta_{4, r}}{N_{4, r}} \frac{\Gamma(3 / 4) r !}{\Gamma(r+3 / 4)}|u|^{r} \\
& <0.83\left(e^{0.99} \sqrt{t^{2}+16}\right)^{r}
\end{aligned}
$$

and

$$
\left|M_{r, 1} A_{r}(1)\right| \leq \frac{\Delta_{4, r}}{N_{4, r}}\left|(i-1) u^{r} \mathcal{X}_{4, r}(w)\right|<0.83 \sqrt{2}\left(e^{0.99} \sqrt{t^{2}+16}\right)^{r} .
$$

Again the first factor of the right hand side of (11) vanishes - this is proved in $\S 3.1$ of [4]. With $|u| \cdot|1-\sqrt{w}|^{2}=\frac{32}{t+\sqrt{t^{2}+16}}<\frac{16}{t}$ and $\arg (w)<\frac{8 t}{t^{2}-16}$, we obtain

$$
\begin{aligned}
\left|M_{r, 0} C_{r}(0)\right| & =\frac{1}{2} \frac{\Delta_{4, r}}{N_{4, r}}\left|\left(i-\beta_{3}\right) u^{r} R_{4, r}(w)\right| \\
& <\frac{5}{t} \frac{\Delta_{4, r}}{N_{4, r}} \frac{\Gamma(r+5 / 4)}{\Gamma(1 / 4) r !}\left(|u| \cdot|1-\sqrt{w}|^{2}\right)^{r}<\frac{1}{t}\left(\frac{e^{2.37}}{t}\right)^{r}
\end{aligned}
$$

and similarly

$$
\left|M_{r, 1} C_{r}(1)\right|<\frac{1.8}{t}\left(\frac{e^{2.37}}{t}\right)^{r} .
$$

Applying now Lemma 7 with $k_{0}=0.83$ ( $0.83 \sqrt{2}$ resp.), $Q=e^{0.99} \sqrt{t^{2}+16}$, $l_{0}=\frac{1}{t}$ ( $\frac{1.8}{t}$ resp.) and $E=t e^{-2.37}$, we obtain lower bounds for $\delta_{3}$ ( $\delta_{2}$ resp.), which together with part a) give the desired bounds for $y$.

Proof of Corollary 2. Suppose that $(x, y)$ with $|x| \leq y$ is a solution of the inequality for some $t \geq 58$. We have $4 \sqrt{\frac{6 t+7}{2 t-1}}<7.03$, thus for $y \geq 8$ we may apply Theorem 2 .

Suppose that $\frac{x}{y}$ is a convergent to $\beta_{2}$. We use Lemma 9.d) to calculate the denominators of the first convergents of $\beta_{2}$ and obtain $y \in\left\{\frac{t}{2}, \frac{t+1}{2}, \frac{t}{2}+1, t+1\right\}$ or $y \geq \frac{t^{2}}{10}-t$, where the last inequality arises from the case $t=2 u$ even. From the first values for $y$ we obtain no solution of our Thue inequality, and the lower bound for $y$ together with Theorem 2.b) gives

$$
\left(\frac{t^{2}}{10}-t\right)^{3-\kappa}<\frac{3.28(6 t+7)}{2.97^{\kappa}}
$$

which is a contradiction for $t \geq 149$. Similarly, if $\frac{x}{y}$ is a convergent to $\beta_{3}$, there is no solution with $y=t$, and $y \geq \frac{t^{2}}{5}-\frac{4 t}{5}+1$ violates the upper bound for $y$ for $t \geq 100$. Next we checked that for $|x| \leq y \leq 7$ we obtain only the solutions stated in Corollary 2, namely

$$
F_{t}^{(4)}(0,1)=1, \quad F_{t}^{(4)}( \pm 1,1)=-4 \quad \text { and } \quad F_{t}^{(4)}( \pm 1,2)= \pm 6 t-7 .
$$

We use Lemma 8 and the same idea as in the proof of Corollary 1 to show that for $58 \leq t \leq 148$ any further solution of the Thue inequality would imply the largest partial quotient in the continued fraction expansion of $\beta_{2}$ (or $\beta_{3}$, resp.) to be larger than

$$
\left(\frac{t^{2}}{10}-t\right)^{2} \frac{2 t-4}{6 t+7}-2>7 t^{2} \quad \text { or } \quad\left(\frac{t^{2}}{5}-\frac{4 t}{5}+1\right)^{2} \frac{t}{6 t+7}-2>19 t^{2} .
$$


Computing the continued fraction expansions until the denominators of the convergents exceed the bounds for $y$ as given by Theorem 2.b), we found as largest partial quotients 15348 for $\beta_{2}$ for $t=78$ and 2486 for $\beta_{3}$ for $t=64$. The necessary computer program was written in MAPLE V running on a PC. This completes our proof.

\section{The SIMPle FAMily of Degree 3}

At last we consider the Thue inequality

$$
\left|F_{t}^{(3)}\right| \leq k(t)
$$

where $k: \mathbb{Z} \rightarrow \mathbb{N}$. For $t \geq 30$ we will give upper bounds for the solutions and discuss it for the special case $k(t)=2 t+3$. For $t \in \mathbb{Z}$ put

$$
P:=F_{t}^{(3)}(X, 1)=X^{3}-t X^{2}-(t+3) X-1=\prod_{i=1}^{3}\left(X-\beta_{i}\right) .
$$

From Lemma 1.b) we know that $\psi_{T_{3}^{2}}: z \mapsto-\frac{1}{z+1}$ permutes the roots $\beta_{i}$ of $P$. The following lemma collects information about $F_{t}^{(3)}$ and $P$, which is analogous to Lemmas 2 and 3 . The proofs are easy and left to the reader.

Lemma 10.

a) The following relations hold:

$$
\begin{gathered}
F_{t}^{(3)}(X, Y)=-F_{t}^{(3)}(-Y, X+Y)=F_{t}^{(3)}(-X-Y, X)=-F_{t}^{(3)}(-X,-Y) \\
=F_{t}^{(3)}(Y,-X-Y)=-F_{t}^{(3)}(X+Y,-X) \\
F_{t}^{(3)}(Y, X)=-F_{-t-3}^{(3)}(X, Y) .
\end{gathered}
$$

b) If $(x, y) \in \mathbb{Z}^{2}$ is a solution of (23), then every pair of the orbit

$$
\{(x, y),(-y, x+y),(-x-y, x),(-x,-y),(y,-x-y),(x+y,-x)\}
$$

is also a solution of (23). Any orbit of non-trivial solutions of (23) contains a pair $(x, y)$ with $y>0$ and $-\frac{y}{2}<x \leq y$.

c) For $t \in \mathbb{Z}, P$ is irreducible over $\mathbb{Q}[X]$, and for $t \geq 4$ the roots of $P$ satisfy the following inequalities:

$$
\begin{aligned}
& t+1+\frac{2}{t}-\frac{3}{t^{2}}<\beta_{1}=\beta<t+1+\frac{2}{t}, \\
& -\frac{1}{t}+\frac{2}{t^{2}}-\frac{3}{t^{3}}<\beta_{2}=-\frac{1}{\beta+1}<-\frac{1}{t}+\frac{2}{t^{2}}-\frac{2}{t^{3}}, \\
& -1-\frac{1}{t}+\frac{1}{t^{2}}<\beta_{3}=-\frac{\beta+1}{\beta}<-1-\frac{1}{t}+\frac{2}{t^{2}} .
\end{aligned}
$$

d) The continued fraction expansion of the root $\beta_{2}$ is

$$
\beta_{2}= \begin{cases}{\left[-1 ; 1, t+1, \frac{t}{2}, 1,3, \ldots\right]} & \text { if } t \geq 16 \text { is even, } \\ {\left[-1 ; 1, t+1, \frac{t+1}{2}, 3,1, \ldots\right]} & \text { if } t \geq 23 \text { is odd } .\end{cases}
$$

In both cases, $-\frac{2 t+3}{2 t^{2}+7 t+10}$ is a convergent to $\beta_{2}$.

Theorem 3. Let $t \geq 1$ and assume that $(x, y) \in \mathbb{Z}^{2}$ is a primitive solution of (23) with $-\frac{y}{2}<x \leq y$ and $\frac{8 k(t)}{2 t+3} \leq y$. 
a) Then $\frac{x}{y}$ is a convergent to $\beta_{2}$, and we have either $y=1$ or

$$
\left|\frac{x}{y}-\beta_{2}\right|<\frac{k(t)}{y^{3}(t+1)} \quad \text { and } \quad y \geq t+2 .
$$

b) Put

$$
\kappa=\frac{\log \left(\sqrt{t^{2}+3 t+9}\right)+0.83}{\log \left(t+\frac{3}{2}\right)-1.3}
$$

If $t \geq 30$ we have

$$
y^{2-\kappa}<17.78 \cdot 2.59^{\kappa} k(t) .
$$

Remark. From Lemma 10.d) we can calculate the denominators of the first convergents of $\beta_{2}$, and obtain the following values: $1, t+2, \frac{t^{2}}{2}+t+1, \frac{t^{2}}{2}+\frac{3 t}{2}+2, \frac{t^{2}}{2}+2 t+3$, $\frac{3 t^{2}+11 t}{2}+8,2 t^{2}+7 t+10$. Since

$$
\left(2 t^{2}+7 t+10\right)^{2-\kappa}<17.78 \cdot 2.59^{\kappa}(2 t+3)
$$

does not hold for $t \geq 10196$, we easily obtain that for these values of $t$ the only primitive solutions of

$$
\left|F_{t}^{(3)}(x, y)\right| \leq 2 t+3
$$

with $-\frac{y}{2}<x \leq y$ are $(0,1),(1,1)$ and $(-1, t+2)$. In a way analogous to Corollaries 1 and 2, one can prove that for $30 \leq t \leq 10195$ there are no further solutions. This result, especially the little computational work needed when using the bounds from Theorem 3.b), should be compared with [15].

Proof of Theorem 3. a) We will only outline the main steps and leave the rest to the reader. Let $(x, y) \in \mathbb{Z}^{2}$ with $-\frac{y}{2}<x \leq y$ be a primitive solution of (23) for some $t \geq 4$. Again we put $\delta_{i}=\left|\frac{x}{y}-\beta_{i}\right|$ and $\delta_{m}=\min \left\{\delta_{i} \mid 1 \leq i \leq 3\right\}$. Since $-\frac{1}{2}<\frac{x}{y} \leq 1$, Lemma 10.c) yields $m=2$. Using the bounds of $\beta_{i}$, we obtain $\delta_{2}<\frac{4 k(t)}{y^{3}(2 t+3)}$, and so $\frac{x}{y}$ must be a convergent to $\beta_{2}$ whenever $\frac{8 k(t)}{2 t+3} \leq y$. If $\frac{x}{y}$ is a convergent to $\beta_{2}$ with $y>1$ we thus have $-\frac{1}{t+2} \leq \frac{x}{y} \leq 0$, from which we derive the upper bound for $\delta_{2}$. For $1 \leq t \leq 3$, we repeat the same ideas using the numerical values of $\beta_{i}$.

b) Let us remark that in (25), $\kappa<2$ only holds for $t \geq 30$, thus we will not consider smaller values of $t$. Taking $U=X^{2}+X+1$, our polynomials $P$ from (24) satisfy the differential equation (7), so we again apply Proposition 1 . We obtain quantities which are very similar to those of Section 5 , e.g. $\quad \lambda=-\frac{3}{4}$, the polynomials $\mathbf{a}, \mathbf{b}, \mathbf{c}, \mathbf{d}$ are $\frac{8}{35}$ times the corresponding polynomials in Section 5 , $\mathbf{u}(0), \mathbf{z}(0)$ are $(-1)$ times the corresponding values in $(20)$, and again, $w=\frac{t-3 \rho^{2}}{t-3 \rho}$. Taking

$$
M_{r}=\left(\frac{9}{2}\right)^{r} \frac{\sqrt{3}}{4} \frac{\Delta_{3, r}}{N_{3, r}} \quad \text { and } \quad u=t-3 \rho,
$$

and using the same arguments as in Section 5, we can show that

$p_{r}=M_{r} B_{r}(0)=2 \Im\left(u^{r} \frac{\Delta_{3, r}}{N_{3, r}} \mathcal{X}_{3, r}(w)\right), \quad q_{r}=M_{r} A_{r}(0)=2 \Im\left(\rho^{2} u^{r} \frac{\Delta_{3, r}}{N_{3, r}} \mathcal{X}_{3, r}(w)\right)$ 
are rational integers with

$$
\begin{gathered}
\left|q_{r}\right|<3.8\left(e^{0.83} \sqrt{t^{2}+3 t+9}\right)^{r} \quad \text { and } \\
\left|q_{r} \beta_{2}-p_{r}\right|<\frac{2.61 \sqrt{3}}{t}\left(\frac{e^{1.3}}{t+3 / 2}\right)^{r} .
\end{gathered}
$$

To obtain these upper bounds, we use Proposition 2.b). Now we again apply Lemma 7, and together with Theorem 3.a), we can obtain the claimed bound (26) in the same way as in the proofs of the previous theorems.

Proof of Corollary 3. This is an immediate consequence of Theorem 3, using

$$
\kappa<1+\frac{2.14}{\log \left(t+\frac{3}{2}\right)-1.3} \quad \text { and } \quad \frac{1}{2-\kappa}<1+\frac{2.14}{\log \left(t+\frac{3}{2}\right)-3.44} .
$$

\section{REFERENCES}

[1] A. Baker, Rational approximations to $\sqrt[3]{2}$ and other algebraic numbers, Quart. J. Math. Oxford 15 (1964), 375-383. MR 30:1977

[2] M. Bennett, Effective measures of irrationality for certain algebraic numbers, J. Austral. Math. Soc. Ser. A 62 (1997), 329-344. MR 98c:11070

[3] Chen Jian Hua, $A$ new solution of the Diophantine equation $X^{2}+1=2 Y^{4}$, J. Number Theory 48 (1994), 62-74. MR 95i:11019

[4] Chen Jian Hua and P. M. Voutier, Complete solution of the Diophantine equation $X^{2}+1=$ $d Y^{4}$ and a related family of quartic Thue equations, J. Number Theory 62 (1997), 71-99. MR 97m:11039

[5] G. V. Chudnovsky, On the method of Thue-Siegel, Ann. of Math. 117 (1983), 325-382. MR 85g:11058

[6] J. H. E. Cohn, Equations with equivalent roots, Acta Arith. 34 (1977), 37-41. MR 56:15554

[7] D. Easton, Effective irrationality measures for certain algebraic numbers, Math. Comp. 46 (1986), 613-622. MR 87f:11047

[8] M. N. Gras, Familles d'unités dans les extensions cycliques réelles de degré 6 de $\mathbb{Q}$, Publ. Math. Fac. Sci. Besançon (1984 - 1986), fasc. 2, 27 pp. MR 88k:11078

[9] A. J. Lazarus, On the class number and unit index of simplest quartic fields, Nagoya Math. J. 121 (1991), 1-13. MR 92a:11129

[10] G. Lettl and A. Pethő, Complete solution of a family of quartic Thue equations, Abh. Math. Sem. Univ. Hamburg 65 (1995), 365-383. MR 96h:11019

[11] G. Lettl, A. Pethö and P. Voutier, On the arithmetic of simplest sextic fields and related Thue equations, Number Theory: Diophantine, Computational and Algebraic Aspects (K. Győry, A. Pethő and V.T. Sós, eds.), Walter de Gruyter Publ. Co., 1998, 331-348.

[12] F. Lorenz, Lineare Algebra II, BI-Wiss. Verlag Mannheim, 1989. MR 90f:15002

[13] K. S. McCurley, Explicit estimates for $\theta(x ; 3, l)$ and $\psi(x ; 3, l)$, Math. Comp. 42 (1984), 265-286. MR 85g:11085

[14] M. Mignotte, Verification of a conjecture of E. Thomas, J. Number Theory 44 (1993), 172-177. MR 94m:11035

[15] M. Mignotte, A. Pethő, F. Lemmermeyer, On the family of Thue equations $x^{3}-$ $(n-1) x^{2} y-(n+2) x y^{2}-y^{3}=k$, Acta Arith. 76 (1996), 245-269. MR 97k:11039

[16] M. Mignotte, A. Pethö, R. Roth, Complete solutions of quartic Thue and index form equations, Math. Comp. 65 (1996), 341-354. MR 96d:11034

[17] A. Pethő, On the resolution of Thue inequalities, J. Symbolic Comput. 4 (1987), 103-109. MR 89b: 11030

[18] Complete solutions to families of quartic Thue equations, Math. Comp. 57 (1991), 777-798. MR 92e: 11023

[19] O. Ramaré and R. Rumely, Primes in arithmetic progressions, Math. Comp. 65 (1996), 397-425. MR 97a:11144 
[20] J. B. Rosser and L. Schoenfeld, Approximate formulas for some functions of prime numbers, Illinois J. Math. 6 (1962), 64-94. MR 25:1139

[21] R. Schoof and L. C. Washington, Quintic polynomials and real cyclotomic fields with large class numbers, Math. Comp. 50 (1988), 543-556. MR 89h:11067b

[22] D. Shanks, The simplest cubic fields, Math. Comp. 28 (1974), 1134-1152. MR 50:4537

[23] E. Thomas, Complete solutions to a family of cubic diophantine equations, J. Number Theory 34 (1990), 235-250. MR 91b:11027

[24] P. M. Voutier, Rational approximations to $\sqrt[3]{2}$ and other algebraic numbers revisited, Indag. Math. (to appear).

Institut für Mathematik, Karl-Franzens-Universität, Heinrichstrasse 36, A-8010 Graz, Austria

E-mail address: guenter.lettl@kfunigraz.ac.at

Department of Mathematics and Informatics, Lajos Kossuth University, P.O. Box 12, H-4010 Debrecen, Hungary

E-mail address: pethoe@math.klte.hu

Department of Mathematics, University of Colorado, Boulder, Colorado 80309

Current address: Optrak Distribution Software Ltd., Cawthorne House, 51 St. Andrew Street, Hertford SG14 1HZ, Great Britain

E-mail address: paul@optrak.co.uk 